\title{
Nanoscale Chemical Features of the Natural Fibrous Material Wood
}

\author{
Claudia Gusenbauer,* Devon S. Jakob, Xiaoji G. Xu,* Dmitri V. Vezenov, Étienne Cabane, \\ and Johannes Konnerth
}

Cite This: Biomacromolecules 2020, 21, 4244-4252

Read Online

\section{ACCESS I \\ Supporting Information}

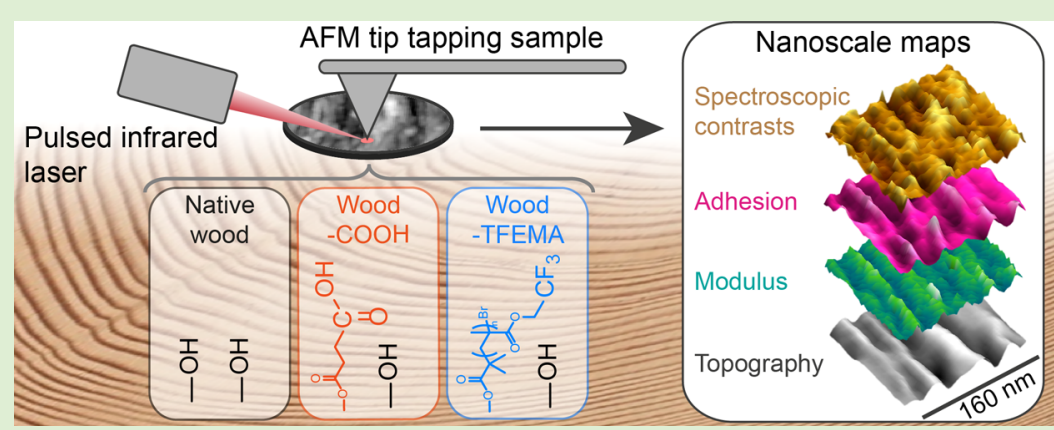

ABSTRACT: Peak force infrared (PFIR) microscopy is a recently developed approach to acquire multiple chemical and physical material properties simultaneously and with nanometer resolution: topographical features, infrared (IR)-sensitive maps, adhesion, stiffness, and locally resolved IR spectra. This multifunctional mapping is enabled by the ability of an atomic force microscope tip in the peak force tapping mode to detect photothermal expansion of the sample. We report the use of the PFIR to characterize the chemical modification of bio-based native and intact wooden matrices, which has evolved into an increasingly active research field. The distribution of functional groups of wood cellulose aggregates, either in native or carboxylated states, was detected with a remarkable spatial resolution of $16 \mathrm{~nm}$. Furthermore, mechanical and chemical maps of the distinct cell wall layers were obtained on polymerized wooden matrices to localize the exact position of the modified regions. These findings shall support the development and understanding of functionalized wood materials.

\section{INTRODUCTION}

The three major components of wood are cellulose, hemicellulose, and lignin, whose distinct allocation and structural arrangement within the lignocellulose scaffold are responsible for the unique properties of wood. ${ }^{1}$ Because the demand of bio-based materials continually increases, the problem of optimal utilization of this renewable resource arises. Chemical and physical modifications broaden the functionality of wood materials and enable applications under demanding conditions such as outdoor or load-bearing applications. ${ }^{2}$ The separation of wooden resources into their components and additional chemical protocols bring further opportunities to create highperformance bio-based polymers. ${ }^{3}$ To promote and support new applications of native or functionalized lignocellulosic materials, material behavior should be tied to fundamental knowledge of structural and chemical features of the raw and processed wood materials. Nanoscopic mapping of chemical composition of a wood cross-section can, in principle, be achieved using scanning probe microscopy (SPM), but common imaging modes do not provide chemical sensitivity. On the other hand, infrared (IR) or Raman imaging is sensitive to composition of organic materials but is limited in resolution by the Abbe-limit. Therefore, direct nanoscale imaging of chemical group distribution in wood structures is difficult without either sample or instrument modifications, and such reports have been limited so far.

A technique that can go beyond the optical diffraction limit of far-field microscopy while preserving the sensitivity to vibrational spectra is atomic force microscopy (AFM)-based IR spectroscopy (AFM-IR). AFM-IR represents an effective, nondestructive approach for characterization at the nanoscale in which spectroscopic surface properties are revealed with high spatial resolution. ${ }^{4}$ The main operating principle behind this method is the induction of thermal expansion of a specimen because of the absorption of IR energy. This expansion is then probed locally by an AFM tip scanning the surface. ${ }^{5,6}$ In cellulose science, for instance, AFM-IR supported improved understanding of polylactic acid nanocellulose composites, ${ }^{7}$ interactions of resins and wood cell walls, ${ }^{8}$ and the chemical composition of pit membranes in wood. ${ }^{9}$

Received: July 6, 2020

Revised: August 26, 2020

Published: August 27, 2020 
(a) Native wood cells

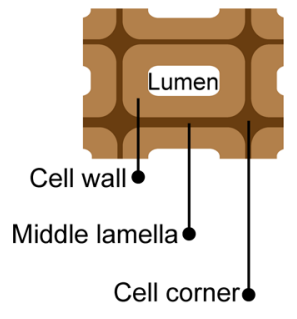

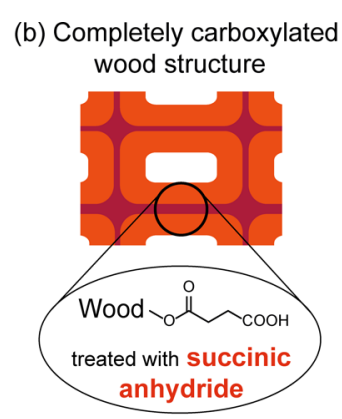

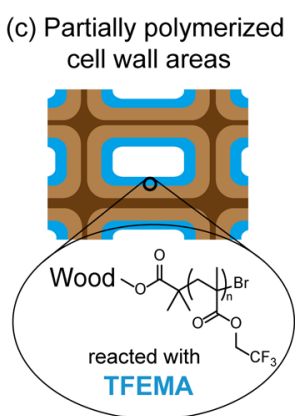

Figure 1. PFIR measurements were carried out on native (a) and chemically modified (b,c) wood structures. (a) Native wood cells consist of cell walls that are built up by a scaffold of cellulose, hemicellulose, and lignin and are connected by the middle lamella. In the dry state, a hollow cavity appears in the center of a wood cell, called lumen. (b) Whole wood structure was esterified by succinic anhydrides leading to an increased amount of carboxylic groups. (c) TFEMA monomers were polymerized onto areas close to the lumen from an initiator previously grafted onto the wood surface.

A further development in combining IR spectroscopy with SPM is represented by peak force IR (PFIR) microscopy, which maps out spectroscopic and mechanical properties simultaneously using an AFM tip tapping the surface of the sample at sub-resonance frequencies and recording photothermal forces arising due to IR laser pulses directed at the sample. ${ }^{10}$ Peak force tapping is performed in the PeakForce Quantitative Nanomechanical Mapping (PeakForce QNM) mode, so that adhesion and modulus values can be extracted from the acquired force-distance curves. Simple PeakForce QNM measurements, without spectroscopic contrast, were applied, for instance, on wooden matrices to locally identify moduli variations ${ }^{11}$ or time-dependent adhesion forces. ${ }^{12}$ With lignocellulose fibers, researchers analyzed the adsorption behavior and morphology of cellulose nanofibers. ${ }^{13,14}$ In PFIR, the addition of an IR laser focused between the AFM tip and the specimen enables simultaneous identification of chemical and mechanical surface variations. A spatial resolution below $10 \mathrm{~nm}$ has been reported for PFIR images of block copolymers, ${ }^{10}$ zymosan particles from yeast, ${ }^{15}$ and source rocks of oil shale. ${ }^{16}$

In this study, we applied the PFIR technique to image ultramicrotomed lignocellulose substrates. We examined chemical surface characteristics and correlated them to nanostructural features for native wood and two functionalized wood materials, which displayed the inherent characteristics superior to native wood materials. One modification procedure was designed for the fabrication of the mechanically stable wastewater filters obtained by treating wood scaffolds with succinic anhydrides. ${ }^{17}$ The second method uses modification with TFEMA (2,2,2-trifluoroethyl methacrylate) polymers to engineer novel, complex wood-based materials. ${ }^{18}$ These substrates were imaged with the aim of achieving spectroscopic contrasts within the lignocellulose nanostructure and help us gain insights into their apparent material properties. We employed IR-sensitive imaging at different wavenumbers and coupled them with adhesion and modulus maps to visualize the distribution of specific functional groups and correlate them with observed mechanical properties. This information was available for examined wooden structures at the spatial resolution down to $16 \mathrm{~nm}$.

\section{MATERIALS AND METHODS}

Spruce wood (Picea abies) was chosen due to its relatively simple and homogeneous wood structure. ${ }^{1}$ Small blocks $\left(1 \times 1 \times 0.5 \mathrm{~cm}^{3}\right)$ were cut out of native spruce wood. Attention was paid to use wood blocks with similar growth ring width of about $1-2 \mathrm{~mm}$ and density of approximately $0.46 \mathrm{~g} / \mathrm{cm}^{3}$. These wood blocks were either tested with no further chemical modification and are referred to here as native wood or were chemically functionalized following two different modification protocols. The first modification process uses succinic anhydride, acetone, and pyridine (anhydrous grade). The second modification process uses TFEMA, $\alpha$-bromoisobutyryl bromide (BiBB), tin(II)-2-ethylhexanoate $\left(\mathrm{Sn}(\mathrm{Oct})_{2}\right)$, copper(II)bromide $\left(\mathrm{Cu}(\mathrm{II}) \mathrm{Br}_{2}\right)$, and $N, N, N^{\prime}, N^{\prime \prime}, N^{\prime \prime}$-pentamethyldiethylenetriamine (PMDETA) dissolved in a mixture of $\mathrm{N}, \mathrm{N}$-dimethylformamide (DMF, anhydrous), pyridine, and methanol. All chemicals were purchased in analytical grade from Sigma-Aldrich (St. Louis, MI, USA), except for pyridine (anhydrous grade), which was purchased from VWR (Radnor, PA, USA). All chemicals were used as received.

2.1. Functionalization of Wood I (Wood-COOH). The modification process that leads to an increased amount of carboxylic groups of all wood cell wall layers (Figure 1b) is described in detail in a previous publication ${ }^{17}$ and results in the material referred to here as wood-COOH. In short, five wooden blocks were dried at $65{ }^{\circ} \mathrm{C}$ in an oven to a constant mass value. Succinic anhydride (reactant) was added in 3 molar equivalent with a wood glucopyranose unit (molecular weight $=162 \mathrm{~g} / \mathrm{mol}$ ) in a flask equipped with a reflux condenser and containing $15 \mathrm{~mL}$ pyridine. The dried wood blocks were immersed in the solution overnight. Subsequently, the solution was heated up to $65{ }^{\circ} \mathrm{C}$ and kept at this temperature for $2 \mathrm{~h}$. Thereafter, the wood cubes were submerged in acetone at room temperature in five cycles before they were dried at $65^{\circ} \mathrm{C}$ in an oven. The weight percentage gain was found to be $15.8 \%$ (calculated on the basis of the dry weights before and after the modification).

2.2. Functionalization of Wood II (Wood-TFEMA). The modification process that leads to growing of poly-TFEMA monomers from the lumen side toward the secondary cell wall (Figure 1c) has also been previously described ${ }^{18}$ and produces the modified wood material referred to here as wood-TFEMA. The technique is based on a surface-initiated activator generated by electron atomic transfer radical polymerization. Five wood cubes underwent Soxhlet extraction, were dried in an oven, and placed in an evacuated Schlenk flask with a septum $\left(10^{-2} \mathrm{mbar}\right)$. A BiBB-pyridine solution [BiBB in 0.5 molar equivalent with a wood glucopyranose unit $($ molecular weight $=162 \mathrm{~g} / \mathrm{mol})]$ was prepared and added to the flask with a syringe. The grafting process lasted $20 \mathrm{~h}$ at room temperature. The cubes were first submerged in methanol and then in acetone and dried at $65{ }^{\circ} \mathrm{C}$. These modified wood blocks (weight percentage gain $=25 \%)$ served as the macroinitiator for the following polymerization step in which poly-TFEMA were grown from the BiBB-modified areas. For this purpose, two flasks were prepared. In one flask, a solution of PMDETA (ligand) and TFEMA (monomer) in DMF was prepared, cooled with ice, and degassed with nitrogen for $1 \mathrm{~h}$. The previously modified wood cubes were placed in the other Schlenk flask containing $\mathrm{Cu}(\mathrm{II}) \mathrm{Br}_{2}$ and equipped with a septum. The flask was subsequently evacuated $\left(10^{-2} \mathrm{mbar}\right), \mathrm{DMF}$ was added, and 

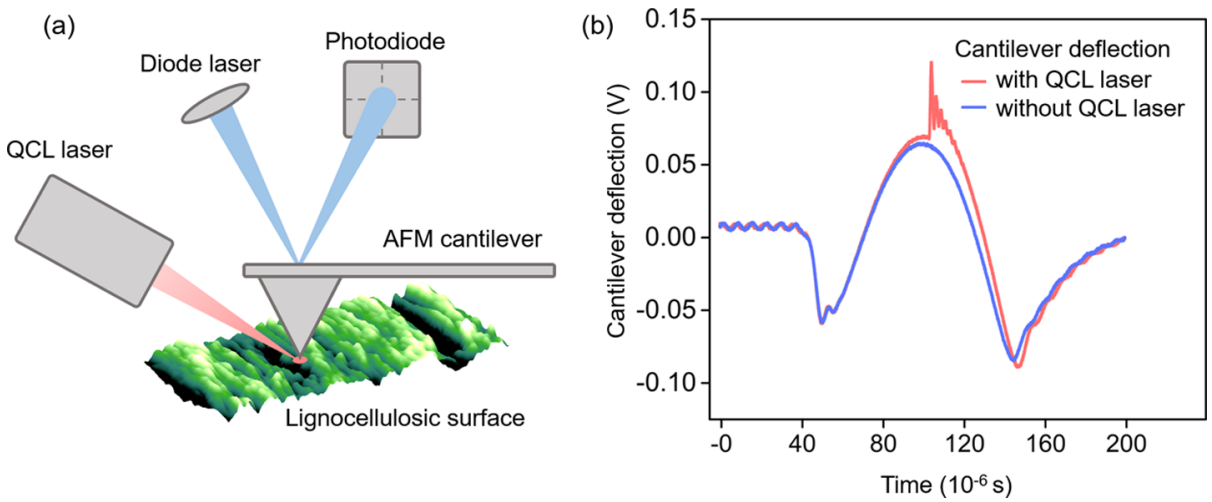

Figure 2. Depiction of the operating principle behind PFIR microscopy. (a) AFM diode laser monitors the deflection of the AFM probe while a QCL induces contact resonances whose characteristics are determined by the properties of the tip-sample contact. (b) Use of the pulsed laser source changes the deflection of the cantilever because of photothermal expansion of the sample surface every other cycle. The subtraction of the cantilever deflection curve measured either with laser-induced changes (red trace) or without laser-induced changes (blue trace) combined with subsequent fast Fourier transform will provide the PFIR signal at this location.

the solution was heated up to $80{ }^{\circ} \mathrm{C}$ in an oil bath. Afterward, the ligand and monomer solution was transferred to the Schlenk flask containing the wood samples, followed by the addition of $\mathrm{Sn}(\mathrm{Oct})_{2}$ (reducing agent) in small steps over $40 \mathrm{~h}$ with a syringe pump. After completion of the reaction, the polymerized wood samples were washed in ethanol, sonicated in ethanol and acetone, and dried at 65 ${ }^{\circ} \mathrm{C}$. The total weight gain was $43 \%$. The molar mass ratios of the chemicals required for modification were calculated, as reported in the previous publication: $[$ TFEMA $] /[$ wood- $\mathrm{BiBB}] /\left[\mathrm{Cu}(\mathrm{II}) \mathrm{Br}_{2}\right] /[\mathrm{PMDE}-$ $\mathrm{TA}] /\left[\mathrm{Sn}(\mathrm{Oct})_{2}\right]=10: 1: 1: 2: 2 \cdot{ }^{18}$

2.3. ATR-FTIR Spectroscopy. Fourier transform IR (FTIR) spectra were acquired with an attenuated total reflectance Fourier transform IR spectroscope (ATR-FTIR, Perkin Elmer Frontier, Waltham, MA, USA). Four scans covering the range from 900 to 1768 $\mathrm{cm}^{-1}$ were acquired for each type of the wood cube (native wood, wood-COOH, and wood-TFEMA). The resolution was set to $2 \mathrm{~cm}^{-1}$. The spectra were averaged, baseline corrected, and normalized to the highest peak with a spectroscopy software (Spectragryph 1.2, Oberstdorf, Germany); the results were visualized with OriginPro 2016 (OriginLab, Northampton, MA, USA).

2.4. Surface Preparation via Ultramicrotoming. To accommodate the limits of the scanning range for the sample heights in the atomic force microscope used for PFIR, smooth surfaces had to be generated. Therefore, small pieces $\left(3 \times 1.5 \times 1.5 \mathrm{~mm}^{3}\right)$ were cut from the samples and glued (Uhu, Uhu plus sofortfest, Bühl/Baden, Germany) onto metal discs. In an ultramicrotome setup (Ultracut-R, Leica, Wetzlar, Germany), the samples were cut with three different diamond knives (Trim 45/Histo/Ultra-AFM; DiATOME, Nidau, Switzerland) consecutively. The radial sections of native wood and wood-COOH were cut and further examined by PFIR. For woodTFEMA samples, transverse cross-sections were cut for the PFIR investigation. The difference between the radial and transverse sections is visualized in the Supporting Information file in Figure S1.

2.5. PFIR Microscopy. The PFIR method is an AFM-based technique for examining surface properties in the peak force tapping mode, also known as the pulsed force mode. Our PFIR instrument was constructed using a MultiMode AFM with Nanoscope V controller (Bruker, Santa Barbara, CA, USA). During the peak force cycle, the oscillating sample driven by a piezo stage (at $2 \mathrm{kHz}$ ) comes into contact with the AFM probe and causes the vertical deflection of the AFM cantilever (platinum-coated silicon probe, nominal spring constant $40 \mathrm{~N} / \mathrm{m}$, tip radius of $\sim 25 \mathrm{~nm}, \mathrm{HQ}: \mathrm{NSC} 15 / \mathrm{Pt}$, MikroMasch, Sofia, Bulgaria). The deflection of the cantilever is monitored by a conventional optical lever detection scheme: a beam from a diode laser, focused at the backside of the cantilever, is reflected to a split photodiode detector, reporting changes in the laser spot position with cantilever bending. As depicted in Figure 2a, the apparatus is extended by a pulsed IR laser [quantum cascade laser
(QCL), MIRcat, Daylight Photonics, California, USA], which is focused between the surface of the sample and the AFM tip using a combination of optical elements. The $100 \mathrm{~ns}$ long pulses are timed to appear every other peak force cycle, changing the deflection versus time response of the cantilever every other peak force cycle because of photothermal expansion of the sample (Figure $2 b$ ). The deflection is digitized and sent to a data acquisition card with a sampling rate of 50 MHz. The PFIR trace is generated by subtracting the cantilever trace without photothermal expansion from the cantilever trace with photothermal expansion. The final PFIR signal is then generated by fast Fourier transform of the PFIR trace and integration of the contact resonance band.

Chemical moieties that are able to absorb more energy from the pulsed laser source will therefore provide a higher PFIR signal. In the point spectrum mode, the tip is fixed to a certain sample position, and the frequency of the QCL laser is swept to obtain a PFIR spectrum similar to a conventional IR spectrum for bulk materials. To obtain IR chemical maps, the laser is fixed to a certain frequency while the tip is scanning the lignocellulose substrates with a scan speed of $0.2 \mathrm{~Hz}$. The elastic modulus and adhesion values can be extracted from the acquired force-distance curves using the Derjaguin-Muller-Toporov model ${ }^{19}$ implemented in the controller software supplied by the instrument manufacturer. ${ }^{16}$ The adhesion force is defined as the maximum force required to break the tip free from the surface contact. The acquired maps were visualized with Gwyddion (freeware, Brno, Czech Republic), and the spectra were plotted with OriginPro 2016. A fully detailed description of the whole PFIR technique can be found in a previous publication. ${ }^{10}$

2.6. Confocal Raman Microscopy. Ultramicrotomed native wood, wood-COOH, and wood-TFEMA in their dry states were analyzed with a confocal Raman microscope (alpha300 RA, WITec $\mathrm{GmbH}$, Ulm, Germany). The distribution of the functional groups after the wood modification was mapped to visualize the chemical composition provided by Raman microscopy. ${ }^{20}$ The spectra were acquired on the transverse cut surface every $300 \mathrm{~nm}$ using a $785 \mathrm{~nm}$ laser (diode laser, $180 \mathrm{~mW}$, CrystaLaser, Reno, NV, USA), an optimized blazed grating $\left(600 \mathrm{~g} \mathrm{~mm}^{-1}\right.$, UHTS spectrometer, Witted Germany), and a deep depletion charge-coupled device camera (Andor, DU401A BR-DD, Oxford Instruments, Abingdon, England) with an integration time of 0.04 to $0.07 \mathrm{~s}$ (wood-TFEMA) and $0.43 \mathrm{~s}$ (wood-COOH). Data analysis together with cosmic ray removal was performed with a WITec Project 4.1 software (WITec GmbH, Ulm, Germany), and Raman images were generated by band integration.

\section{RESULTS AND DISCUSSION}

3.1. PFIR Mapping of Native Wood Structures. Capturing data from multiple channels of the PFIR apparatus, we imaged the topography alongside chemical and mechanical 
(a) Height [nm]

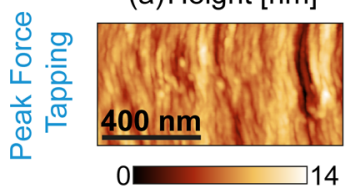

(b) Adhesion [nN]

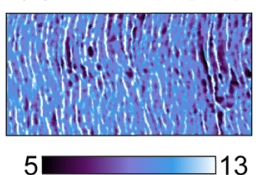

(c) Modulus [GPa]

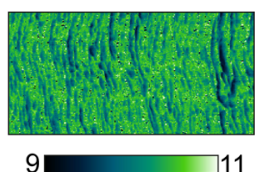

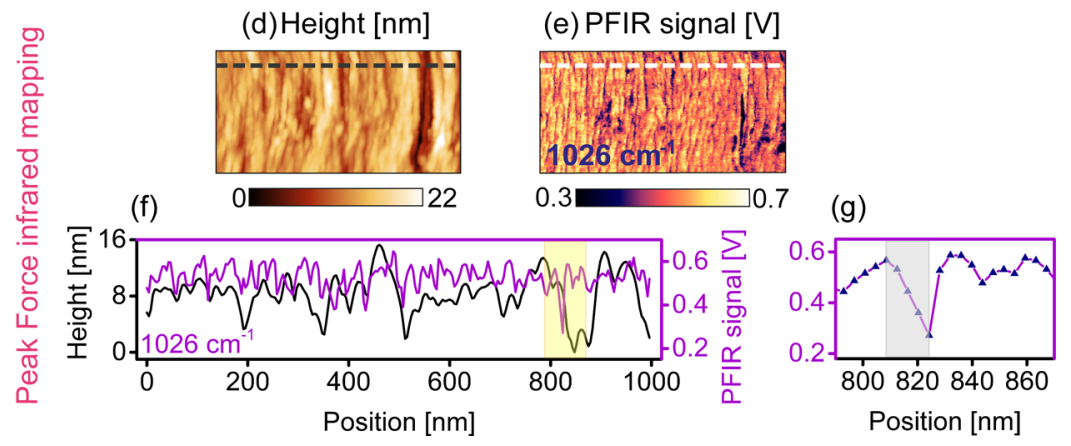

Figure 3. Application of the PFIR principle to the native, radial wood sections. By scanning an ultramicrotomed wood cell wall, topography (a,d), adhesion (b), and modulus (c) maps of the secondary wood cell wall were recorded. (e) Corresponding PFIR map was obtained by focusing an IR laser between the tapping AFM tip and the surface of the sample. The laser was tuned to $1026 \mathrm{~cm}^{-1}$, characteristic of C-O bond stretching. (f) Cross-section of the PFIR image [indicated by the white line in (e)] traces the distribution of cellulosic polymers and compared to a cross-section of the height image [indicated by the black line in (d)]. (g) Spatial resolution of $16 \mathrm{~nm}$ (defined as the full width of a stepped feature) is observed from a cross-section shown in yellow box in (f).

features of an ultramicrotomed native wood cell wall. Figure 3 is obtained by setting the scan rate to $0.1 \mathrm{~Hz} /$ line and the probe oscillation amplitude to $30 \mathrm{~nm}$. The scanning area was chosen on the thickest wood cell wall, called secondary (S2) cell wall, where the fibrous scaffold could be visualized (Figure 3a). The diameters of these scanned fibrous structures were found to be within $30-40 \mathrm{~nm}$ - extracted profiles are depicted in Figure S2 in the Supporting Information. Additionally, the adhesion and Young's modulus were mapped at the same position (Figure 3b,c). We observed average adhesion forces of $9.4 \mathrm{nN}$ with maximum adhesion forces up to $14.0 \mathrm{nN}$ on areas between the wood fibrils. The average modulus was found to be $10.3 \mathrm{GPa}$ and was slightly higher in valleys between the fibrils than at the top of the fibrils. A 3D-depiction of the fibrous topography with adhesion and modulus overlays is illustrated in Figure S3 in the Supporting Information.

After the modulus and adhesion maps were recorded without the application of the IR laser, the laser was turned on and tuned to $1026 \mathrm{~cm}^{-1}$ to identify areas correlated to C$\mathrm{O}$ stretching ${ }^{21}$ in the same scan area. Figure $3 \mathrm{e}$ displays a typical spectroscopic contrast of the selected surface area in the wood cell wall because of the ability of PFIR to detect differences in photothermal expansion by the AFM tip. We observed that most regions of the scanned area showed PFIR response with only some domains that did not show any response at the selected wavenumber. Higher PFIR signals are displayed in brighter colors and revealed the different local magnitudes of PFIR responses attributed to $\mathrm{C}-\mathrm{O}$ stretching. A 3D-depiction of the fibrous topography with PFIR overlay at $1026 \mathrm{~cm}^{-1}$ is shown in Figure S3 in the Supporting Information. Figure $3 \mathrm{f}$ superimposes profiles extracted at the same position from the height and PFIR images (lines in Figure 3d,e), which allowed identifying PFIR-sensitive areas of the fibrous wood structure more precisely. Therefore, the PFIR signal can be assigned to the nanoscopic fibrous structures, approximately correlating with the topography, while generally revealing much finer features. For example, a spatial resolution of $16 \mathrm{~nm}$ was revealed from a cross-section in the PFIR map
(Figure $3 g$ ), measured as the width of a steep edge profile. ${ }^{16}$ Note that a shift in the topography images is visible when comparing Figure 3a,d, which was due to the use of the openloop scanner in our AFM.

The fibrous geometry that is visible in Figure 3 originates from the hierarchical wood ultrastructure. In this scaffold, small cellulose chains are aligned to microfibrils (diameter $<5 \mathrm{~nm}$ ), which in turn form thicker aggregates called cellulose aggregates or cellulose fibrils. ${ }^{22}$ These cellulose aggregates are further embedded in a mixture of lignin and hemicellulose. $^{23}$ The fibril diameters of $30-40 \mathrm{~nm}$ observed in our study agree well with the diameter values of cellulose aggregates reported in the literature in which measurements using transmission electron microscopy of spruce wood found diameters between 20 and $40 \mathrm{~nm} .^{2,24}$ Nanostructural analysis of wood cell walls revealed that the diameters naturally vary between 10 and $100 \mathrm{~nm}^{25}$ and depend on the position within the cell wall. ${ }^{26}$

Adhesion phenomena have been investigated in wood scaffolds and found to be influenced by the wood roughness. ${ }^{27,28}$ Comparisons of absolute values found in literature remained unreliable because of the differences in measurement modes and settings used as well as variations in the chemical functionalities of AFM tips. Because native wood materials are hygroscopic and the wood surface readily binds water, our measurements, performed in ambient air, might be highly influenced by capillary forces between the AFM tip and the wood surface. ${ }^{29}$ Assuming perfect wetting of both tip and sample surfaces (contact angle $\theta=0^{\circ}$ ), we expect to observe forces on the order of $F=4 \pi R \gamma \cos \theta \approx 23 \mathrm{nN}$ ( $\gamma$ is the surface tension of water), within a factor of 1.5-2 of our experimental values. We therefore only consider variations within the same adhesion map, where our main observation is that stickier areas occur between the fibrils. This observation is potentially a consequence of the differences in the effective local radius of curvature $\left(1 / R=1 / R_{\text {tip }}+1 / R_{\text {sample }}\right)$; areas that are flatter or have negative curvature (such as valleys) should display greater adhesion. 
(a) Native wood
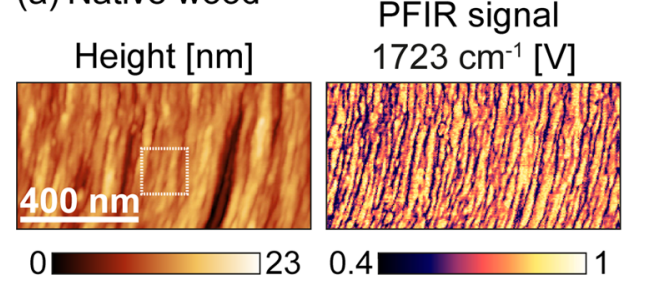

(c)ATR-FTIR

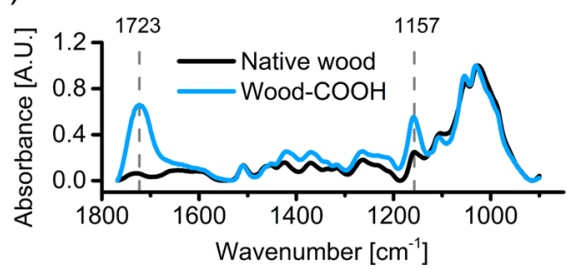

(b) Wood-COOH

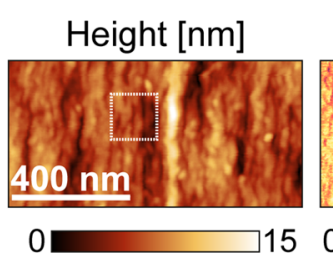

150.4

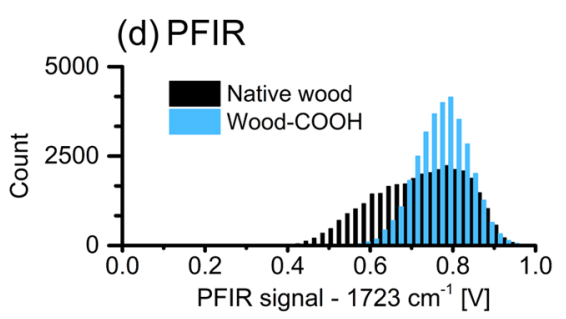

Figure 4. PFIR images of native (a) and carboxylated (wood-COOH) (b) radial wood sections taken at $1723 \mathrm{~cm}^{-1}$, which is characteristic for $\mathrm{C}=$ $\mathrm{O}$ stretching, reveal the changes in the distribution of carboxyl groups. (c) Conventional FTIR spectra of macroscopic samples of native and carboxylated wood. (d) Histograms of the PFIR intensities at $1723 \mathrm{~cm}^{-1}$ of the corresponding PFIR maps in (a,b). White squares in (a,b) show the position of the PFIR maps in Figure 5.

The modulus of bulk spruce wood structures at the macroscale level is found to be $11 \mathrm{GPa}$ (flexural modulus of elasticity of P. abies). ${ }^{30}$ Comparing moduli values determined with different AFM techniques, researchers observed moduli of wood cross-sections of $3.2 \mathrm{GPa}$ of the secondary cell wall obtained in the Quantitative Imaging mode. ${ }^{31}$ Additionally, the study of the effect of the fibril orientation on indentation moduli of spruce wood found a decrease of modulus with increasing indentation angles (from $19 \mathrm{GPa}$ for an indentation angle of $0^{\circ}$ to $6 \mathrm{GPa}$ for an indentation angle of $90^{\circ}$ with respect to the microfibril axis). ${ }^{32}$ The moduli values presented in Figure 3 depict similar magnitudes of modulus, but the anisotropy and natural variability of wood materials ${ }^{1}$ have still to be considered when comparing absolute values. The influence of the microfibril angle on the mechanical strength also needs to be accounted for in further studies to allow the comparison of specific stiffness values. ${ }^{33}$

Because we observed a high IR absorbance at $1026 \mathrm{~cm}^{-1}$ in the conventional ATR-FTIR spectrum (Figure 4c), the first PFIR maps were generated at that wavenumber. This band is assigned to $\mathrm{C}-\mathrm{O}$ stretching of primary alcohols ${ }^{34}$ as in mainly cellulose. ${ }^{35}$ In Figure 3f, we can match the PFIR signal and location of the fibril structure at the nanoscale. Lower signals were observed between the fibrous features, and higher signals were measured in the center of the fibrous structures, that is, cut-open cellulose aggregates. This observation is in accordance with the model where cellulose aggregates are surrounded by a different component, for example, lignin, which acts as a coating of the aggregates visualized by lower PFIR signals between the aggregates. ${ }^{36}$ Furthermore, features in the PFIR images were smaller by a factor of $2-3$ compared to the corresponding height image, which gives evidence to features with smaller diameters, presumably the cellulose microfibrils.

Full spectra could not be acquired due to the fact that two neighboring pixels often showed varying signals (inhomogeneous distribution of wood components) complicated by the use of the open-loop AFM system in which the AFM probe position in the image plane is not completely constant.

3.2. Chemical Surface Changes within Esterified Wood Structures. In addition to analyzing native scaffolds, we evaluated the success of chemical modification procedures by visualizing compositional changes at the cell wall level with PFIR. The treatment of the wood samples with succinic anhydride resulted in an increased amount of carboxylic groups within the wood matrix (wood-COOH, see Section 2.2). This modification procedure aims at adding to wood the function of removing copper ions from waste water. ${ }^{17}$ The increase in carboxylic groups was verified on three levels. First, conventional ATR-FTIR measurements were acquired on native and modified specimens (Figure 4c); an increased IR absorbance at $1723 \mathrm{~cm}^{-1}\left(\mathrm{C}=\mathrm{O}\right.$ vibration) and at $1157 \mathrm{~cm}^{-1}(\mathrm{C}-\mathrm{O}$ stretching $)^{17}$ confirmed the increase of $-\mathrm{COOH}$ functional groups after modification.

Second, Raman maps at $1728 \mathrm{~cm}^{-1}$ were acquired on the cross-sections of native wood and wood-COOH to track the carboxyl group content. These scans revealed an overall increase of $-\mathrm{COOH}$ groups within all cell wall layers of samples treated with succinic anhydride (results are depicted in Figure S4 in the Supporting Information). Lastly, we overcame the fundamental limitations of the ultimate resolution in light microscopy techniques (Rayleigh criterion or diffraction limit) and were able to map native and modified wood surfaces with the PFIR setup at the spatial resolution far better than other light-based spectroscopic techniques. Similar to Figure 3, ultramicrotomed radial sections of the secondary cell wall area were scanned with the IR laser tuned at 1723 $\mathrm{cm}^{-1}$ to show the change in the chemical composition at the surface (compare normalized PFIR maps in Figure 4a,b). Features depleted or enriched in $\mathrm{COOH}$ functionality (absorbing at $1723 \mathrm{~cm}^{-1}$ ) with sizes on the order of 10-20 $\mathrm{nm}$ are clearly resolved. Additionally, histograms highlighted the overall increase of PFIR signal intensities at $1723 \mathrm{~cm}^{-1}$ of the wood-COOH samples (Figure $4 \mathrm{~d}$ ) at the expense of the low-intensity regions of native wood.

PFIR maps at $1723 \mathrm{~cm}^{-1}$ of both surfaces, native wood and wood-COOH, showed an energy absorbance at that wavenumber $(\mathrm{C}=\mathrm{O}$ stretching), resulting in thermal expansion. The PFIR absorbance at native wood surfaces can be attributed to the carbonyl group of glucuronic acid, which appears in xylans of hemicelluloses. ${ }^{37}$ Comparing Figure $4 a, b$, we can observe that almost all structures in wood-COOH 
showed uniformly high PFIR signal originating from wood cell wall esterified by succinic anhydride. ${ }^{17}$ A zoom into the cell wall areas for both samples (Figure 5) helps with the (a)
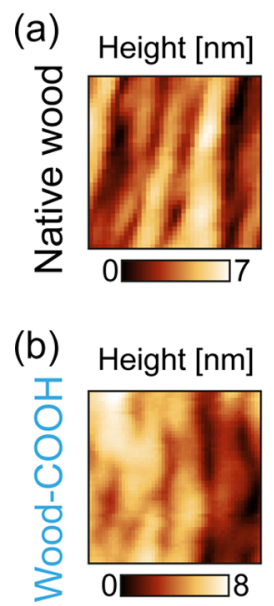

PFIR signal

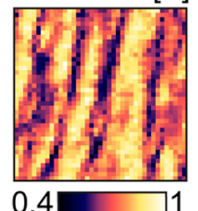

$0.4 \square 1$

\section{PFIR signal}
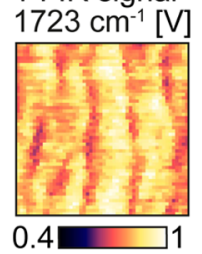

$1723 \mathrm{~cm}^{-1}[\mathrm{~V}$ ] Modulus [GPa]
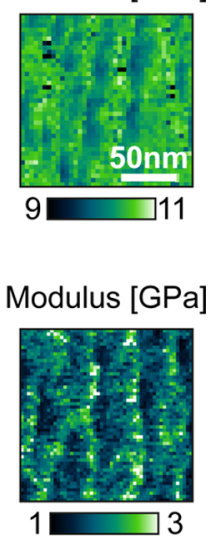

Figure 5. Zoom into the cell wall of native (a) and chemically treated (b) wood samples reveals the distribution of elastic moduli and PFIR signals with high spatial resolution. Wood-COOH structures possessed higher PFIR intensities at $1723 \mathrm{~cm}^{-1}$ but lower moduli compared to the native wood scaffolds.

localization of the regions of high PFIR intensities and stiffness. Thus, we observed that the top of the fibers possessed higher PFIR intensities, while these areas also possessed lower moduli characteristics. This combination of high $\mathrm{COOH}-$ content and low stiffness might result from the composition of the wood cell wall: as described previously, cellulose molecules generate microfibrils surrounded by a lignin-hemicellulose compound matrix. These microfibrils align with larger structures, which are embedded in the matrix material. ${ }^{22,38}$ $7-10 \%$ of the hemicelluloses in spruce wood are built up by arabinoglucuronoxylan, including gluronic acid, ${ }^{33}$ which could be responsible for the high PFIR intensities on the fibril structures. In wood-COOH samples, the hydroxyl groups of cellulose were esterified with succinic anhydride, resulting in an increased amount of carboxyl groups, which then cause high PFIR intensities of the whole cellulose aggregates in wood$\mathrm{COOH}$ samples. This carboxylation is accompanied by the dramatic drop (by a factor of 4-5) in Young's modulus.

3.3. Chemical Identification of Polymerized Wood Cells. The polymerization with TFEMA leads to a partial polymerization several micrometers deep in certain cell wall areas. ${ }^{18}$ The cross-section of a TFEMA polymerized cell wall was scanned in the PFIR mode, so that different cell layers, that is, middle lamella, cell corner, secondary cell wall, and polymerized cell wall, could be identified (Figure 6a). The scanning position was selected, so that deep lumina, that is, hollow cavities in the middle of the cell, were not scanned because they could exceed the $z$-limit of the AFM scan range. The modulus map of the scanned section illustrated that different cell wall layers could be easily distinguished by different magnitudes of elastic modulus (Figure 6b). The secondary cell wall possessed highest stiffness of 5.0 GPa, followed by the polymerized cell wall area (3.2 GPa), the middle lamella $(2.6 \mathrm{GPa})$, and the cell corner $(2.5 \mathrm{GPa})$; these values were extracted by the mask tool in the Gwyddion software. A reversed order was observed for PFIR intensities when scanning the same area with the IR laser tuned to 1278 $\mathrm{cm}^{-1}$ : the cell corner showed the highest PFIR intensities, followed by the polymerized cell wall area and the middle lamella, while the secondary cell wall possessed the lowest PFIR intensities. Tuning the laser to $1743 \mathrm{~cm}^{-1}$, we found a similar trend, with the difference that the polymerized wood cell wall area possessed the highest PFIR intensity. Because both mechanical properties and absorbance influence photothermal expansion (which serves as the key parameter in generating spectroscopic contrasts), it is expected that certain areas with similar moduli, for example, cell corner or polymerized cell wall, could show different relative PFIR intensities at different wavenumbers (comparing typical PFIR maps in Figure $6 b-d)$. Therefore, areas with a specific
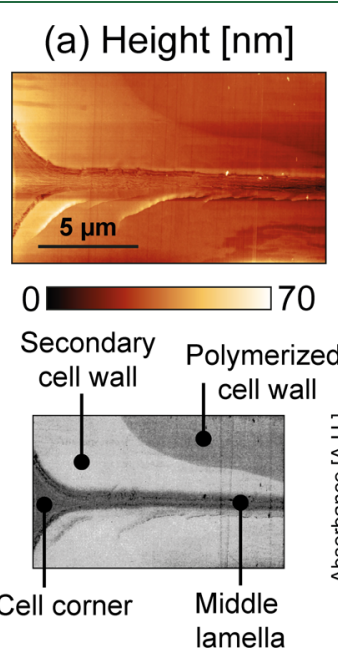

(b) Modulus [GPa]

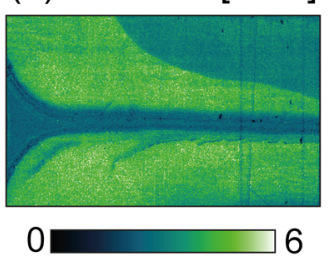

(e) IR spectra

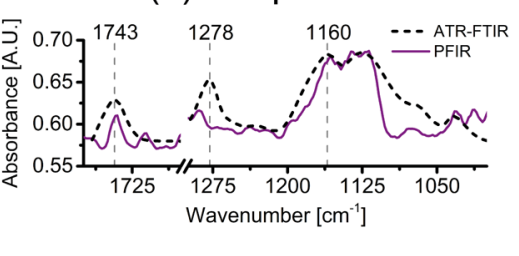

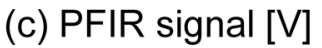

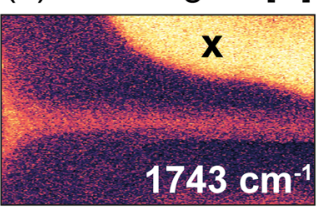

Or

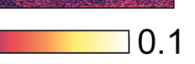

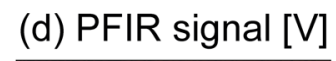

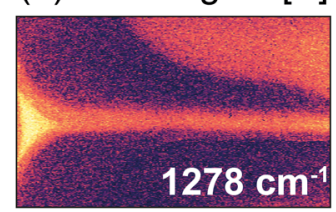

0

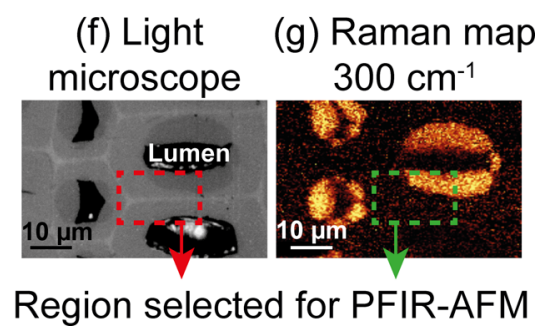

Figure 6. Application of the PFIR principle on polymerized wood cross-sections. The scan parameters were set to 512 samples per line, a set-point of $10 \mathrm{nN}$, and a scan rate of $0.14 \mathrm{~Hz}$. (a, top) Position of the PFIR mapping was chosen, so that the cell wall and middle lamella of two neighboring cell walls were scanned, as indicated on the light microscopy image of the same area (a, bottom). Polymerized areas were identified in PFIR maps taken at 1743 (c) and $1278 \mathrm{~cm}^{-1}$ (d) alongside moduli scans (b). Cell wall areas partially polymerized with TFEMA were identified prior to PFIR measurements with light (f) and Raman (g) microscopy. (e) Typical PFIR point spectrum taken from a polymerized area indicated by a cross in (c) is compared to ATR-FTIR spectrum of TFEMA-modified samples. 
modulus showed different PFIR intensities at changed laser wavelengths, which demonstrated that PFIR signals could be coupled to but are not solely originating from the differences in stiffness.

A point spectrum was acquired at the polymerized area, which showed a similar trace when compared to the conventional FTIR spectra (Figure 6e). Peaks at $1278 \mathrm{~cm}^{-1}$, assigned to overlapping signals of $\mathrm{C}-\mathrm{F}$ stretching vibration of the introduced polymer ${ }^{39}$ and aromatic $\mathrm{C}-\mathrm{O}$ stretching of lignin, ${ }^{40}$ and peaks at $1743 \mathrm{~cm}^{-1}$, assigned to $\mathrm{C}=\mathrm{O}$ stretching vibration of the ester carbonyl group, ${ }^{18,41}$ were present in both types of spectra. Additionally, the characteristic cellulose peaks ${ }^{37}$ around $1160 \mathrm{~cm}^{-1}$ were detected. Prior to PFIR analysis, Raman maps were acquired at the TFEMA-specific band at $476 \mathrm{~cm}^{-1}$ (Figure S5 in the Supporting Information) or at the BiBB-specific band at $300 \mathrm{~cm}^{-1}$ (Figure $6 \mathrm{~g}$ ) to identify the location of the polymerized modified cell wall areas. The PFIR maps were subsequently taken on the exactly same wood cell wall after removing several thin sections a few micrometers thick to eliminate possible effects of the exposure to the high-power Raman laser.

Reviewing Raman and PFIR maps, we observed that both methods revealed that the main regions of the polymerized wood cell wall are located around the cell wall lumen, which is a frequent observation in wood polymerization procedures. ${ }^{18,42,43}$ Modification chemicals are mainly transported via lumina within the wood structure, thus, producing the higher degree of modification closer to lumen areas. In contrast to Raman microscopy, we can match chemical signals and topographical features in PFIR with high resolution and at superior signal-to-noise ratio. Distinct boundaries between cell walls are readily apparent in the PFIR images and are clearly chemically modified with the polymerization procedure employed here. The strong PFIR signal at $1278 \mathrm{~cm}^{-1}$ in the middle lamella derives from $\mathrm{C}-\mathrm{O}$ stretching of aromatic molecular structures of lignin but might give evidence that some polymerization took place in the middle lamella region. Maps of Young's modulus acquired simultaneously with the PFIR data agreed well with the conventional AFM studies on wood cell wall substrates. Previous research also found that the secondary cell wall was stiffer than the middle lamella. ${ }^{11}$ In that study, PeakForce QNM measurements of the secondary cell wall determined the moduli values of $21.3 \mathrm{GPa}$. The much lower stiffness values, compared to native wood, seen in Figure $6 \mathrm{~b}$, are likely the consequence of the chemical processing of our samples under harsh conditions that lowered the general density and stiffness of the functionalized wood material (also observed here for wood-COOH samples). In addition, the applied chemical modification of the wood matrix could cause a decrease in the mechanical strength due to swelling, dissolution, or depolymerization of the wood cell wall components. $^{33}$

\section{CONCLUSIONS}

We applied PFIR microscopy to analyze native and chemically modified wood. The fibrous wood structure was mapped to identify topographical, mechanical, and chemical features of native, carboxylated, or polymerized cell wall regions. Exploiting thermal expansion of the surface induced by a pulsed laser system of the PFIR apparatus at a specific wavelength, we demonstrated chemically sensitive imaging of wood samples in their dry state with nanometer scale (10-20 $\mathrm{nm})$ resolution.
The distribution of specific functional groups was determined, and spectra were collected from polymerized wood scaffolds. We observed that not only areas around the lumen but also the middle lamella showed indication of the introduced polymer modification. This information could be extracted from the PFIR images, while it was missing from the Raman data due to the fundamental limitations of far-field optical microscopy. These points will require further investigation, and PFIR can provide a way to correlate nanoand microstructural characterization of the changes in the chemical composition with observed changes in the macroscopic behavior.

Besides the high spatial resolution derived in a nondestructive manner, there are several practical benefits of the PFIR mapping technique in the field of wood characterization: (i) simple sample preparation, (ii) insensitivity to fluorescence (compared to, e.g., Raman microscopy), (iii) high signal-tonoise ratio, and (iv) no need of staining steps, chemical modification of the AFM probes, or requirement for imaging under liquid (e.g., as in the case of chemical force microscopy $\left.{ }^{29}\right)$. With the experimental setup used in this study, the current limitations in spatial resolution of light microscopy methods could be exceeded to open the door to routine, information-rich characterization of the ultrastructural and chemical surface properties of lignocellulose materials. Coupled with the quantitative results on macroscopic properties, this approach to chemically sensitive imaging could become a major tool in the rational design of wood materials with improved or new functionality.

\section{ASSOCIATED CONTENT}

\section{Supporting Information}

The Supporting Information is available free of charge at https://pubs.acs.org/doi/10.1021/acs.biomac.0c01028.

Explanation of transverse and radial cutting direction; determination of wood fiber diameter; and 3D presentation of PFIR maps, additional Raman maps of native, modified wood structures (PDF)

\section{AUTHOR INFORMATION}

\section{Corresponding Authors}

Claudia Gusenbauer - Institute of Wood Technology and Renewable Materials, Department of Materials Sciences and Process Engineering, BOKU-University of Natural Resources and Life Sciences Vienna, 3430 Tulln, Austria; orcid.org/ 0000-0003-3520-3732; Email: claudia.gusenbauer@ boku.ac.at

Xiaoji G. Xu - Department of Chemistry, Lehigh University, Bethlehem, Pennsylvania 18015, United States; orcid.org/ 0000-0003-0847-5871; Email: xgx214@lehigh.edu

\section{Authors}

Devon S. Jakob - Department of Chemistry, Lehigh University, Bethlehem, Pennsylvania 18015, United States; 0 orcid.org/ 0000-0002-6534-4803

Dmitri V. Vezenov - Department of Chemistry, Lehigh University, Bethlehem, Pennsylvania 18015, United States

Étienne Cabane - Institute for Building Materials, ETH Zürich, 8093 Zürich, Switzerland; EMPA-Swiss Federal Laboratories for Materials Science and Technology, 8600 Dübendorf, Switzerland; 이이.org/0000-0001-5613-8816 
Johannes Konnerth - Institute of Wood Technology and Renewable Materials, Department of Materials Sciences and Process Engineering, BOKU-University of Natural Resources and Life Sciences Vienna, 3430 Tulln, Austria; 10 orcid.org/ 0000-0003-3826-8566

Complete contact information is available at:

https://pubs.acs.org/10.1021/acs.biomac.0c01028

\section{Author Contributions}

C.G. and D.S.J. performed the PFIR measurements. C.G. prepared the samples, performed IR measurements and data analyzes, and prepared figures and text of the manuscript. É.C. performed the functionalization experiments on wood samples. X.G.X. und D.V.V. supervised the experimental part of the joint project. J.K. supervised the experimental part of the joint project and supported writing the manuscript. All authors reviewed the manuscript.

\section{Notes}

The authors declare no competing financial interest.

\section{ACKNOWLEDGMENTS}

Financial support is gratefully acknowledged by the Austrian Science Fund FWF (project number: I 2247), the Lower Austrian Research and Education Society NFB (project number: SC16-004), the Swiss National Science Foundation SNSF (project number: 160041), and the Austrian Marshall Plan Foundation. We thank Notburga Gierlinger from BOKU-Institute of Biophysics for performing the Raman microscopy measurements. Additionally, we gratefully acknowledge Julien Jaxel for supporting the sample preparation. We thank William Leon and Craig Pointer for supporting the practical work in the laboratory.

\section{REFERENCES}

(1) Fengel, D.; Wegener, G. Wood Chemistry, Ultrastructure, Reactions; Kessel Verlag: Remagen, 2003; pp 26-27.

(2) Hill, C. A. Wood Modification: Chemical, Thermal and Other Processes; John Wiley \& Sons: Chichester, 2006; p 20.

(3) Dufresne, A. Nanocellulose: From Nature to High Performance Tailored Materials; Walter de Gruyter GmbH \& Co. KG: Berlin, 2012; p 321.

(4) Dazzi, A.; Prater, C. B. AFM-IR: Technology and Applications in Nanoscale Infrared Spectroscopy and Chemical Imaging. Chem. Rev. 2017, 117, 5146-5173.

(5) Hammiche, A.; Pollock, H. M.; Reading, M.; Claybourn, M.; Turner, P. H.; Jewkes, K. Photothermal FT-IR spectroscopy: A step towards FT-IR microscopy at a resolution better than the diffraction limit. Appl. Spectrosc. 1999, 53, 810-815.

(6) Anderson, M. S. Infrared spectroscopy with an atomic force microscope. Appl. Spectrosc. 2000, 54, 349-352.

(7) Nair, S. S.; Chen, H.; Peng, Y.; Huang, Y.; Yan, N. Polylactic Acid Biocomposites Reinforced with Nanocellulose Fibrils with High Lignin Content for Improved Mechanical, Thermal, and Barrier Properties. ACS Sustainable Chem. Eng. 2018, 6, 10058-10068.

(8) Wang, X.; Deng, Y.; Li, Y.; Kjoller, K.; Roy, A.; Wang, S. In situ identification of the molecular-scale interactions of phenol-formaldehyde resin and wood cell walls using infrared nanospectroscopy. RSC Adv. 2016, 6, 76318-76324.

(9) Pereira, L.; Flores-Borges, D. N. A.; Bittencourt, P. R. L.; Mayer, J. L. S.; Kiyota, E.; Araújo, P.; Jansen, S.; Freitas, R. O.; Oliveira, R. S.; Mazzafera, P. Infrared Nanospectroscopy Reveals the Chemical Nature of Pit Membranes in Water-Conducting Cells of the Plant Xylem. Plant Physiol. 2018, 177, 1629-1638.
(10) Wang, L.; Wang, H.; Wagner, M.; Yan, Y.; Jakob, D. S.; Xu, X. G. Nanoscale simultaneous chemical and mechanical imaging via peak force infrared microscopy. Sci. Adv. 2017, 3, No. e1700255.

(11) Arnould, O.; Siniscalco, D.; Bourmaud, A.; Le Duigou, A.; Baley, C. Better insight into the nano-mechanical properties of flax fibre cell walls. Ind. Crops Prod. 2017, 97, 224-228.

(12) Frybort, S.; Obersriebnig, M.; Müller, U.; Gindl-Altmutter, W.; Konnerth, J. Variability in surface polarity of wood by means of AFM adhesion force mapping. Colloids Surf., A 2014, 457, 82-87.

(13) Zhu, C.; Soldatov, A.; Mathew, A. P. Advanced microscopy and spectroscopy reveal the adsorption and clustering of $\mathrm{Cu}$ (ii) onto TEMPO-oxidized cellulose nanofibers. Nanoscale 2017, 9, 74197428.

(14) Frone, A. N.; Berlioz, S.; Chailan, J.-F.; Panaitescu, D. M. Morphology and thermal properties of PLA-cellulose nanofibers composites. Carbohydr. Polym. 2013, 91, 377-384.

(15) Li, W.; Wang, H.; Xu, X. G.; Yu, Y. Simultaneous Nanoscale Imaging of Chemical and Architectural Heterogeneity on Yeast Cell Wall Particles. Langmuir 2020, 36, 6169-6177.

(16) Jakob, D. S.; Wang, L.; Wang, H.; Xu, X. G. SpectroMechanical Characterizations of Kerogen Heterogeneity and Mechanical Properties of Source Rocks at $6 \mathrm{~nm}$ Spatial Resolution. Anal. Chem. 2019, 91, 8883-8890.

(17) Vitas, S.; Keplinger, T.; Reichholf, N.; Figi, R.; Cabane, E. Functional lignocellulosic material for the remediation of copper(II) ions from water: Towards the design of a wood filter. J. Hazard. Mater. 2018, 355, 119-127.

(18) Vidiella del Blanco, M.; Gomez, V.; Keplinger, T.; Cabane, E.; Morales, L. F. G. Solvent-Controlled Spatial Distribution of SI-AGETATRP Grafted Polymers in Lignocellulosic Materials. Biomacromolecules 2019, 20, 336-346.

(19) Derjaguin, B.; Muller, V.; Toporov, Y. On different approaches to the contact mechanics. J. Colloid Interface Sci. 1980, 73, 293-294.

(20) Gierlinger, N.; Keplinger, T.; Harrington, M. Imaging of plant cell walls by confocal Raman microscopy. Nat. Protoc. 2012, 7, 16941708.

(21) Türker-Kaya, S.; Huck, C. A review of mid-infrared and nearinfrared imaging: principles, concepts and applications in plant tissue analysis. Molecules 2017, 22, 168.

(22) Fahlén, J.; Salmén, L. Cross-sectional structure of the secondary wall of wood fibers as affected by processing. J. Mater. Sci. 2003, 38, $119-126$.

(23) Frey-Wyssling, A. The ultrastructure of wood. Wood Sci. Technol. 1968, 2, 73-83.

(24) Singh, A. P.; Daniel, G. The S2 Layer in the Tracheid Walls of Picea abies Wood: Inhomogeneity in Lignin Distribution and Cell Wall Microstructure. Holzforschung 2001, 55, 373-378.

(25) Booker, R. E.; Sell, J. The nanostructure of the cell wall of softwoods and its functions in a living tree. Holz Roh-Werkst. 1998, $56,1-8$.

(26) Donaldson, L. Cellulose microfibril aggregates and their size variation with cell wall type. Wood Sci. Technol. 2007, 41, 443-460.

(27) Colson, J.; Andorfer, L.; Nypelö, T. E.; Lütkemeier, B.; Stöckel, F.; Konnerth, J. Comparison of silicon and OH-modified AFM tips for adhesion force analysis on functionalised surfaces and natural polymers. Colloids Surf., A 2017, 529, 363-372.

(28) Jin, X.; Kasal, B. Adhesion force mapping on wood by atomic force microscopy: influence of surface roughness and tip geometry. $R$. Soc. Open Sci. 2016, 3, 160248.

(29) Noy, A.; Vezenov, D. V.; Lieber, C. M. Chemical Force Microscopy. Annu. Rev. Mater. Sci. 1997, 27, 381-421.

(30) Niemz, P.; Sonderegger, W. Holzphysik: Physik des Holzes und der Holzwerkstoffe; Carl Hanser Verlag GmbH Co KG: München, 2017; p 297.

(31) Casdorff, K.; Keplinger, T.; Burgert, I. Nano-mechanical characterization of the wood cell wall by AFM studies: comparison between AC- and QI mode. Plant Methods 2017, 13, 60.

(32) Jäger, A.; Hofstetter, K.; Buksnowitz, C.; Gindl-Altmutter, W.; Konnerth, J. Identification of stiffness tensor components of wood cell 
walls by means of nanoindentation. Composites, Part A 2011, 42, 2101-2109.

(33) Rowell, R. M. Handbook of Wood Chemistry and Wood Composites; CRC Press: Boca Raton, 2013, p 48.

(34) Socrates, G. Infrared and Raman Characteristic Group Frequencies: Tables and Charts; John Wiley \& Sons: Chichester, 2004. (35) Pandey, K. K. A study of chemical structure of soft and hardwood and wood polymers by FTIR spectroscopy. J. Appl. Polym. Sci. 1999, 71, 1969-1975.

(36) Adobes-Vidal, M.; Frey, M.; Keplinger, T. Atomic force microscopy imaging of delignified secondary cell walls in liquid conditions facilitates interpretation of wood ultrastructure. J. Struct. Biol. 2020, 211, 107532.

(37) Stevanic, J. S.; Salmén, L. Orientation of the wood polymers in the cell wall of spruce wood fibres. Holzforschung 2009, 63, 497-503.

(38) Page, D. A note on the cell-wall structure of softwood tracheids. Wood Fiber Sci. 1976, 7, 246-248.

(39) Estillore, N. C.; Advincula, R. C. Free-Standing Films of Semifluorinated Block Copolymer Brushes from Layer-by-Layer Polyelectrolyte Macroinitiators. Macromol. Chem. Phys. 2011, 212, $1552-1566$.

(40) Hergert, H. L. Infrared spectra of lignin and related compounds. II. Conifer lignin and model compounds1, 2. J. Org. Chem. 1960, 25, 405-413.

(41) Chen, Y.; Chen, D.; Ma, Y.; Yang, W. Multiple levels hydrophobic modification of polymeric substrates by UV-grafting polymerization with TFEMA as monomer. J. Polym. Sci., Part A: Polym. Chem. 2014, 52, 1059-1067.

(42) Gusenbauer, C.; Cabane, E.; Gierlinger, N.; Colson, J.; Konnerth, J. Visualization of the Stimuli-responsive Surface Behavior of Functionalized Wood Material by Chemical Force Microscopy. Sci. Rep. 2019, 9, 18569.

(43) Cabane, E.; Keplinger, T.; Künniger, T.; Merk, V.; Burgert, I. Functional lignocellulosic materials prepared by ATRP from a wood scaffold. Sci. Rep. 2016, 6, 31287. 